\title{
Ensaio de Taxa de Secagem e Escore de Defeitos para a Predição da Qualidade da Secagem Convencional da Madeira de Eucalyptus sp. - Parte 2
}

\author{
Djeison Cesar Batista ${ }^{1}$, Ricardo Jorge Klitzke², Márcio Pereira da Rocha ${ }^{2}$, Tharcia \\ Ribeiro Batista ${ }^{1}$
}

${ }^{1}$ Departamento de Ciências Florestais e da Madeira, Universidade Federal do Espírito Santo - UFES, Jerônimo Monteiro/ES, Brasil

${ }^{2}$ Departamento de Engenharia e Tecnologia Florestal, Universidade Federal do Paraná - UFPR, Curitiba/PR, Brasil

\begin{abstract}
RESUMO
Este trabalho é a continuação da pesquisa de Klitzke \& Batista (2010), e teve como objetivo principal avaliar se a qualidade da madeira na metodologia da secagem drástica a $100{ }^{\circ} \mathrm{C}$ corresponde àquela da secagem convencional. Utilizou-se madeira de 11 anos de Eucalyptus saligna, Eucalyptus grandis e Eucalyptus dunnii, que foram secas conjuntamente em uma câmara-piloto convencional, realizando-se três repetições. Os defeitos de secagem avaliados foram rachaduras de superfície e de topo, encanoamento e colapso, dos quais foi produzido um escore médio por espécie, que foi comparado com o escore de defeitos do ensaio de secagem drástica, e também com a densidade básica e a contração volumétrica total da madeira. Eucalyptus grandis e Eucalyptus saligna tiveram a melhor qualidade de secagem. Foi rejeitada a hipótese de se poder utilizar o escore de defeitos do ensaio de secagem drástica como forma de prever a qualidade da secagem convencional das espécies estudadas.
\end{abstract}

Palavras-chave: eucalipto, secagem drástica a $100^{\circ} \mathrm{C}$, proposta de metodologia.

\section{Tests of Drying Rate and Scoring of Defects for the Prediction of Conventional Kiln Drying Quality of Eucalyptus sp. Wood - Part 2}

\begin{abstract}
This research is a continuation of Klitzke \& Batista (2010) work. The main objective was to assess whether the wood quality in the methodology of drastic drying test at $100{ }^{\circ} \mathrm{C}$ corresponds to that of conventional kiln drying. It was used 11 years old Eucalyptus saligna, Eucalyptus grandis and Eucalyptus dunnii woods, which were kiln dried together in the same batch in a pilot scale kiln, doing three repetitions. Drying defects evaluated were end and surface cracks, cupping and collapse, which generated a mean score by species, which was compared to the score of defects of the drastic drying test, as well as to the basic density and the total volumetric shrinkage. Eucalyptus grandis and Eucalyptus saligna had higher wood quality in conventional drying than Eucalyptus dunnii. It was rejected the hypothesis of using the score of defects in drastic drying test as a way of predicting the quality of conventional kiln drying.
\end{abstract}

Keywords: eucalypt, drastic drying at $100^{\circ} \mathrm{C}$, proposed methodology. 


\section{INTRODUÇÃO}

A secagem é, indiscutivelmente, umas das etapas mais importantes do processamento primário da madeira sólida na indústria madeireira. Apesar disso, do ponto de vista científico, a secagem da madeira tem pouca representatividade no cenário brasileiro.

Isso pode ser explicado pela dificuldade prática em se empreender a pesquisa aplicada nessa área. Por exemplo, existem alguns empecilhos para o desenvolvimento de trabalhos em escala industrial: o grande volume das câmaras, o que dificulta o controle das variáveis do processo, o que é importante para um material heterogêneo como a madeira; os equipamentos industriais normalmente possuem capacidade limitada de mecanismos de controle, como a quantidade de pinos sensores, o que é prejudicial em termos de pesquisa, embora seja adequada para o controle industrial; a laboriosidade da amostragem da madeira a ser avaliada; a insalubridade das câmaras de secagem e das atividades associadas ao setor; a dificuldade em se divulgar resultados obtidos em empresas, por causa do protecionismo natural dos empresários, em especial os programas de secagem, que são considerados sigilosos.

A pesquisa em escala laboratorial no Brasil tem sido desenvolvida primariamente pelas universidades e centros especializados, que são instituições públicas, na maioria dos casos. Para tanto, é necessário um ferramental básico, dentre eles, uma câmara-piloto de pequena capacidade (até quatro metros cúbicos), o que não está disponível em todos os casos, pois é um equipamento de elevado custo. Mesmo se tratando de um equipamento de pequena capacidade, comparado aos industriais, ainda assim é necessário um volume de madeira maior do que o normalmente utilizado em outras pesquisas na área de Tecnologia e Utilização de Produtos Florestais, o que, da mesma maneira, nem sempre está disponível ao pesquisador.

Para solucionar esses problemas de ordem prática e ferramental, alguns pesquisadores têm buscado desenvolver, validar e aprimorar pesquisas em escala laboratorial que possam reproduzir o comportamento da secagem convencional em escala industrial (Andrade et al., 2001; Barbosa et al., 2005; Batista et al., 2012; Ciniglio, 1998; Mattos et al., 1999; Santos, 2002). Estes autores têm seguido a mesma linha da pesquisa pioneira de Brandão (1989), que estudou a metodologia proposta por Terazawa (1965), o qual preconizou que pequenas amostras de madeira submetidas à secagem drástica apresentam defeitos em nível proporcional aos que ocorreriam durante a secagem convencional.

Com base nessa metodologia, os pesquisadores brasileiros têm submetido pequenas amostras de madeira à secagem a $100^{\circ} \mathrm{C}$ em estufas de esterilização, e obtido as curvas de secagem das espécies, além de informações para a elaboração de programas de secagem, tais como temperatura inicial, temperatura final e potencial de secagem.

Contudo, a maioria dos resultados obtidos em escala laboratorial não tem sido comprovada na prática, haja vista as dificuldades mencionadas anteriormente. Klitzke \& Batista (2010) utilizaram a metodologia da secagem drástica a $100^{\circ} \mathrm{C}$, associada a um método de caracterização de escores de defeitos, para a predição da qualidade da secagem convencional na madeira de Eucalyptus grandis, Eucalyptus saligna e Eucalyptus dunnii. No entanto, os resultados obtidos ainda carecem de validação em secagem convencional.

No presente estudo, que é a continuação do trabalho de Klitzke \& Batista (2010), o objetivo principal foi avaliar se a qualidade da madeira na metodologia da secagem drástica a $100{ }^{\circ} \mathrm{C}$ (medida em termos de escores de defeitos), corresponde àquela da secagem convencional. O objetivo secundário foi avaliar a qualidade das madeiras de Eucalyptus saligna, Eucalyptus grandis e Eucalyptus dunnii submetidas às mesmas condições de secagem convencional.

\section{MATERIAL E MÉTODOS}

\subsection{Caracterização do material}

Estudou-se a madeira de Eucalyptus saligna, Eucalyptus grandis e Eucalyptus dunnii coletada de plantios clonais homogêneos de 11 anos de idade, plantados em espaçamento 2,5 $\times 2,5$ metros, oriundos de Telêmaco Borba, Paraná. De cada espécie foi utilizada a primeira tora (três metros) de 15 árvores com diâmetro à altura do peito maior ou igual a 20 centímetros, fuste retilíneo e aparentemente sadias. Utilizaram-se tábuas tangenciais retiradas da porção intermediária do raio formado entre a medula e a casca, de dimensões nominais iniciais de $30 \times 150 \times 3.000 \mathrm{~mm}$. 
O estudo de Klitzke \& Batista (2010) foi realizado com este mesmo material, assim, o trabalho foi conduzido com base na premissa do conhecimento prévio das propriedades físicas (densidade e contração total), taxa de secagem e escore de defeitos do ensaio de secagem drástica a $100{ }^{\circ} \mathrm{C}$.

\subsection{Preparação e controle das cargas de secagem}

De cada tábua original foram serradas três tábuas curtas de $745 \mathrm{~mm}$ de comprimento, que foram utilizadas na secagem convencional em câmara-piloto com capacidade nominal de quatro metros cúbicos.

Foram secas três cargas, em que cada uma correspondeu a uma pilha com dimensões aproximadas de $745 \mathrm{~mm}$ de largura, $500 \mathrm{~mm}$ de altura e $1.350 \mathrm{~mm}$ de comprimento, composta por 81 tábuas curtas $(745 \mathrm{~mm}$ de comprimento), livres de defeitos (nós e medula, principalmente), 27 de cada espécie, resultando em nove camadas de madeira, cada uma com nove tábuas. As tábuas foram empilhadas sobre um vagonete, com o comprimento no sentido transversal ao fluxo de ar da câmara e separadas por tabiques de $25 \mathrm{~mm}$ de seção e $1.350 \mathrm{~mm}$ de comprimento. As tábuas das diferentes espécies foram distribuídas homogeneamente, havendo tábuas de todas as espécies distribuídas pelas camadas da pilha.

Para o controle da secagem foram utilizadas as tábuas mais pesadas de cada lote, em que foram fixados dois pares de pinos sensores por tábua (um par de pinos curtos e outro de pinos longos). Utilizaram-se duas tábuas de Eucalyptus dunnii, uma na parte anterior da primeira camada e outra na parte posterior da última camada; uma tábua de Eucalyptus saligna, na porção mediana da quarta camada; e outra de Eucalyptus grandis, na porção mediana da sexta camada. Foram utilizadas mais tábuas-controle de Eucalyptus dunnii porque se esperava que esta espécie fosse de mais difícil secagem dentre as avaliadas, por causa da maior densidade básica média $\left(0,56 \mathrm{~g} \cdot \mathrm{cm}^{-3}\right)$.

As três espécies foram secas conjuntamente, para que fossem submetidas às mesmas condições de secagem e, assim, permitisse uma avaliação igualitária da qualidade e comparação com o ensaio de secagem drástica a $100^{\circ} \mathrm{C}$.

$\mathrm{Na}$ Tabela 1 encontra-se o programa de secagem que foi utilizado nas três cargas, que foram secas até a umidade final de $10 \%$, com velocidade do ar constante de 2,0 m.s. $\mathrm{s}^{-1}$ ao longo de todo o ciclo.

\subsection{Avaliação da secagem convencional}

Essa avaliação foi feita de acordo com a ocorrência de defeitos, após 24 horas do término da fase de resfriamento das cargas de secagem, conforme recomendação de Galvão \& Jankowsky (1985), em que foram analisados as rachaduras de superfície, rachaduras de topo, encanoamento e colapso. Os defeitos foram classificados em "presente" ou "ausente", utilizando-se

Tabela 1. Programa de secagem.

Table 1. Drying schedule.

\begin{tabular}{|c|c|c|c|c|c|c|}
\hline Umidade(\%) & $\operatorname{TBS}\left({ }^{\circ} \mathrm{C}\right)$ & $\mathrm{TBU}\left({ }^{\circ} \mathrm{C}\right)$ & UR(\%) & UE(\%) & Tempo (h) & PS \\
\hline Aquecimento & 40 & 39 & 94 & - & 3 & - \\
\hline 45 & 40 & 38 & 88 & 18 & - & 2,5 \\
\hline 35 & 44 & 41 & 84 & 16 & - & 2,2 \\
\hline 31 & 46 & 42 & 78 & 14 & - & 2,2 \\
\hline 28 & 50 & 45 & 77 & 14 & - & 2,0 \\
\hline 25 & 54 & 48 & 71 & 12 & - & 2,1 \\
\hline 20 & 58 & 49 & 64 & 10 & - & 2,0 \\
\hline 17 & 60 & 49 & 55 & 8 & - & 2,1 \\
\hline 15 & 62 & 49 & 48 & 7 & - & 2,1 \\
\hline 12 & 66 & 51 & 46 & 6 & - & 2,0 \\
\hline 10 & 66 & 47 & 35 & 5 & - & 2,0 \\
\hline Uniformização - 10 & 64 & 56 & 66 & 10 & 8 & - \\
\hline Condicionamento - 10 & 62 & 56 & 73 & 12 & 8 & - \\
\hline Resfriamento -10 & 44 & 35 & 55 & 9 & 4 & - \\
\hline
\end{tabular}

TBS: temperatura de bulbo seco; TBU: temperatura de bulbo úmido; UR: umidade relativa; UE: umidade de equilíbrio do ambiente; PS: potencial de secagem. 
$100 \%$ das tábuas. Os demais tipos de empenamentos não foram avaliados por causa do comprimento reduzido das tábuas. A classificação qualitativa da porcentagem de defeitos de secagem foi realizada de acordo com o proposto por Martins (1984) citado por Brandão (1989), que faz cinco discriminações: ausente (0-10\%), pequena (11-30\%), moderada (31-50\%) e grande (51-100\%).

\subsection{Análise estatística}

Contaram-se as tábuas para cada um dos quatro tipos de defeitos de secagem avaliados, e com base no somatório foi produzido apenas um escore por espécie. Os escores foram analisados de acordo com o teste não paramétrico $\mathrm{H}$ de Kruskal-Wallis, em nível de significância de 5\%. Este teste fornece um método não paramétrico para a análise de variância (ANOVA) para classificação de um critério ou experimentos com um fator, podendo-se fazer generalizações (Spiegel, 1994). Os testes não paramétricos são aplicados quando a população analisada não apresenta distribuição normal, como no caso das contagens.

\section{RESULTADOS E DISCUSSÃO}

\subsection{Apresentação dos resultados de Klitzke \& Batista (2010)}

Os principais resultados que serviram de base para o presente trabalho foram apresentados para facilitar a discussão, e eliminar o inconveniente da consulta ao artigo.

\subsubsection{Propriedades físicas}

Com base nas propriedades físicas foi concluído que a espécie Eucalyptus grandis oferecia maior perspectiva de apresentar melhor qualidade de secagem, seguida por Eucalyptus saligna e Eucalyptus dunnii. Isto porque a menor densidade básica absoluta $\left(0,45 \mathrm{~g} \cdot \mathrm{cm}^{-3}\right)$ e a menor contração volumétrica total $(14,10 \%)$ foram daquela espécie, indicando menor propensão a defeitos de secagem decorrentes da anisotropia de contração, além de maior facilidade de movimentação da água na madeira. Em contraposição encontra-se o Eucalyptus dunnii, com a maior densidade básica $\left(0,56\right.$ g. $\left.\mathrm{cm}^{-3}\right)$ e maior contração volumétrica total $(18,71 \%)$. A contração volumétrica total do Eucalyptus saligna foi intermediária às demais espécie $(15,17 \%)$, ao passo que a densidade básica não diferiu estatisticamente do Eucalyptus grandis $\left(0,46\right.$ g.cm $\left.{ }^{-3}\right)$.

\subsubsection{Ensaio de secagem drástica a $100^{\circ} \mathrm{C}$}

A taxa de secagem $\left(\mathrm{g} / \mathrm{cm}^{2} . \mathrm{h}\right)$ não foi uma boa ferramenta para predição da qualidade da secagem convencional, porque as médias não diferiram entre as espécies, impossibilitando qualquer associação. No entanto, o escore dos defeitos avaliados (colapso, rachaduras de superfície, internas e de topo) foi uma boa ferramenta, porque apresentou a mesma tendência das propriedades físicas, ou seja, quanto mais densa e instável fosse a madeira, maior seria o escore de defeitos.

Na Figura 1 encontram-se os resultados por espécie do escore de defeitos do ensaio de secagem drástica a $100{ }^{\circ} \mathrm{C}$, contudo, foi realizada uma nova abordagem da apresentação dos resultados. No trabalho de Klitzke \& Batista (2010) foi concluído, de acordo com o teste de Kruskal-Wallis ( $\mathrm{H}$ calculado $=18,70^{*}$ ), que houve diferença significativa entre os escores de defeitos das espécies. Porém, não foi realizada análise para saber quais espécies diferiram entre si. Conforme apresentado no gráfico de caixas (boxplot) na Figura 1, o maior escore de defeitos foi do Eucalyptus dunnii, ao passo que as demais espécies não diferiram estatisticamente entre si.

Dessa forma, foi possível obter mais informações dos resultados, pela nova abordagem da análise, e pode-se chegar a uma nova conclusão, de que o comportamento do escore de defeitos no ensaio de secagem drástica

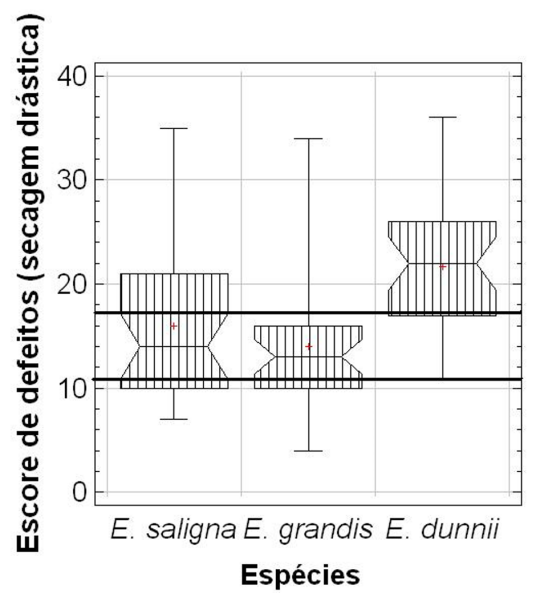

Figura 1. Escore de defeitos do ensaio de secagem drástica, por espécie.

Figure 1. Score of defects of the drastic drying test, by species. 
seguiu a mesma tendência dos resultados de densidade básica. Esta informação será importante para embasar pesquisas futuras a respeito da mesma metodologia.

\subsection{Defeitos da secagem convencional}

Na Tabela 2 encontram-se os resultados por espécie da porcentagem das tábuas com defeitos de secagem convencional, e a classificação entre parênteses refere-se ao critério qualitativo proposto por Martins (1984) citado por Brandão (1989). De cada espécie foram avaliadas todas as 81 tábuas, que foram secas nas três cargas, ou seja, 27 tábuas por espécie por carga.

As espécies tiveram classificação "ausente" quanto às rachaduras de topo e de superfície, mesma daquela observada por Ciniglio (1998) na secagem de Eucalyptus grandis e Eucalyptus urophylla. No entanto, 7,91\% das tábuas de Eucalyptus grandis tiveram rachaduras de topo, superior aos 5,6\% obtidos por Mellado (1993). Para o Eucalyptus dunnii, este defeito ocorreu em 9,88\% das tábuas, semelhante aos $10 \%$ obtidos no programa de secagem mais suave utilizado por Severo (1998).

Quanto ao encanoamento, a porcentagem de defeitos das espécies foi classificada como "grande", e foram superiores aquelas obtidas por outros autores (Ciniglio, 1998; Mellado, 1993; Severo, 1998). Este resultado deve-se principalmente a dois fatores: i) a instabilidade dimensional da madeira, predominantemente de lenho juvenil, com elevados coeficiente de anisotropia e contração volumétrica total e; ii) utilização de peças tangenciais, que são naturalmente mais propensas a este tipo de empenamento do que as radiais.

Com relação ao colapso, as espécies receberam classificações diferentes, de acordo com o critério utilizado. Os 55,56\% das tábuas de Eucalyptus dunnii com colapso foram superiores aos $35 \%$ do programa de secagem mais suave analisado por Severo (1998), porém, inferior aos $67,5 \%$ e $87,5 \%$ dos outros dois programas avaliados pelo mesmo autor, de maior potencial de secagem. Tais resultados corroboram com a classificação ("grande") deste defeito nesta espécie, indicando que a etapa de secagem inicial (remoção da água capilar) deve ser mais suave do que a utilizada. Os $35,80 \%$ de tábuas com colapso do Eucalyptus grandis foram superiores aos 8,3\% de Ciniglio (1998), corroborando com a classificação ("moderada") da incidência deste defeito nesta espécie. O Eucalyptus saligna foi a única espécie em que a ocorrência de colapso foi classificada como "pequena". A despeito dos resultados, nos casos em que se necessita de madeira de melhor qualidade, podem ser utilizadas fases iniciais de secagem mais suaves e técnicas de recuperação de colapso para a minimização deste defeito (Mellado, 1993; Severo, 1998).

\subsection{Escore de defeitos da secagem convencional}

De acordo com o teste $\mathrm{H}$ a 5\% de significância $\left(\mathrm{H}\right.$ calculado $\left.=0,91^{\text {ns }}\right)$ não houve diferença significativa entre os escores de defeitos da secagem convencional das espécies. Este resultado foi considerado ruim para a metodologia proposta, porque se esperava que este escore tivesse o mesmo comportamento, em termos de análise estatística, do escore de defeitos da secagem drástica a $100^{\circ} \mathrm{C}$.

Por exemplo, considere-se uma câmara de secagem carregada com 4.200 peças de $30 \times 150 \times 3.000 \mathrm{~mm}$, resultando em $56,7 \mathrm{~m}^{3}$ de madeira empilhada. Ao analisar-se o colapso (Tabela 2), que é um dos defeitos e secagem mais graves, porque altera drasticamente a forma das peças, reduzindo muito o volume aproveitável

Tabela 2. Porcentagem de tábuas com defeitos de secagem convencional por espécie.

Table 2. Percentage of boards with conventional kiln drying defects by species.

\begin{tabular}{|c|c|c|c|c|c|}
\hline \multirow[b]{2}{*}{ Espécie } & \multicolumn{4}{|c|}{$(\%)$} & \multirow[b]{2}{*}{ Total* } \\
\hline & $\begin{array}{c}\text { Rachaduras de } \\
\text { topo }\end{array}$ & $\begin{array}{c}\text { Rachaduras de } \\
\text { superfície }\end{array}$ & Encanoamento & Colapso & \\
\hline Eucalyptus saligna & $\begin{array}{c}4,94 \\
\text { (ausente) }\end{array}$ & $\begin{array}{c}3,70 \\
\text { (ausente) }\end{array}$ & $\begin{array}{c}67,90 \\
\text { (grande) }\end{array}$ & $\begin{array}{c}28,40 \\
\text { (pequena) }\end{array}$ & 104,94 \\
\hline Eucalyptus grandis & $\begin{array}{c}7,91 \\
\text { (ausente) }\end{array}$ & $\begin{array}{c}0 \\
\text { (ausente) }\end{array}$ & $\begin{array}{c}60,49 \\
\text { (grande) }\end{array}$ & $\begin{array}{c}35,80 \\
(\text { moderada })\end{array}$ & 103,70 \\
\hline Eucalyptus dunnii & $\begin{array}{c}9,88 \\
\text { (ausente) }\end{array}$ & $\begin{array}{c}4,94 \\
\text { (ausente) }\end{array}$ & $\begin{array}{c}74,07 \\
\text { (grande) }\end{array}$ & $\begin{array}{c}55,56 \\
\text { (grande) }\end{array}$ & 144,45 \\
\hline
\end{tabular}

${ }^{*}$ A soma das porcentagens de defeitos ultrapassa $100 \%$ porque uma mesma tábua apresentou mais de um tipo de defeito. 
(até mesmo inutilizando completamente a madeira), os 55,56\% das peças de Eucalyptus dunnii resultariam em um volume de $31,5 \mathrm{~m}^{3}$; ao passo que para Eucalyptus saligna e Eucalyptus grandis este volume seria de 16,1 e $20,3 \mathrm{~m}^{3}$, respectivamente.

Portanto, analisando-se economicamente os prejuízos da secagem nesse caso, não se pode negar que as duas últimas espécies tiveram melhor qualidade de secagem, embora os escores médios não tenham diferido estatisticamente. Essa discussão reforça a ideia de a metodologia utilizada não ter sido adequada para as espécies analisadas, não se atingindo a hipótese inicial da pesquisa. Na Figura 2 encontram-se as linhas de tendência dos escores de defeitos de ambas as metodologias.

Conforme apresentado na Figura 2, notam-se para ambas as metodologias que os escores tiveram a mesma tendência entre as espécies, ou seja, o Eucalyptus grandis teve menos defeitos, seguido por Eucalyptus saligna e Eucalyptus dunnii. Contudo, conforme discutido anteriormente, os escores médios da secagem convencional não foram estatisticamente diferentes.

Acredita-se que a metodologia não tenha sido validada porque os escores médios da secagem convencional foram gerados com base na quantidade (contagem) de tábuas por tipo de defeito avaliado (colapso, encanoamento, rachaduras de topo e de superfície). Assim, para pesquisas futuras, sugere-se que o escore médio seja produzido pelo escore da avaliação individual de cada tábua, da mesma forma que o realizado na metodologia da secagem drástica (Klitzke \& Batista, 2010). Também, sugere-se que em ambas as metodologias sejam avaliados os mesmos tipos de defeitos.

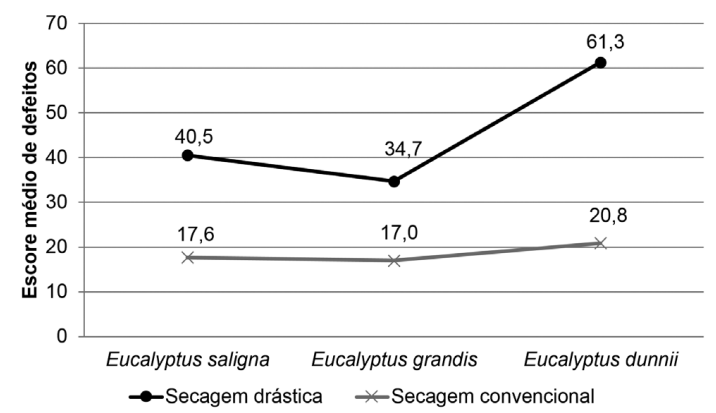

Figura 2. Linhas comparativas dos escores médios do ensaio de secagem drástica e secagem convencional.

Figure 2. Comparative lines of mean scores of drastic drying test and conventional kiln drying.

\section{CONCLUSÕES}

Com base na nova análise dos dados de Klitzke \& Batista (2010), comprovou-se que o comportamento do escore de defeitos no ensaio de secagem drástica a $100{ }^{\circ} \mathrm{C}$ seguiu a mesma tendência dos resultados de densidade básica.

Para a mesma condição de secagem, as espécies Eucalyptus grandis e Eucalyptus saligna obtiveram maior qualidade na secagem convencional do que o Eucalyptus dunnii. Este resultado está diretamente associado com a densidade e a contração volumétrica total.

Foi rejeitada a hipótese de se poder utilizar o escore de defeitos do ensaio de secagem drástica a $100^{\circ} \mathrm{C}$ como forma de prever a qualidade da secagem convencional das espécies estudadas, com base na metodologia utilizada. Contudo, para pesquisas futuras, acredita-se que a hipótese ainda possa ser validada pela modificação da metodologia de obtenção do escore de defeitos da secagem convencional.

\section{AGRADECIMENTOS}

Ao Conselho Nacional de Desenvolvimento Científico e Tecnológico, pela concessão de bolsa ao primeiro autor.

\section{STATUS DA SUBMISSÃO}

Recebido: 13 jun., 2013

Aceito: 12 set., 2015

AUTOR(ES) PARA CORRESPONDÊNCIA

\section{Djeison Cesar Batista}

Departamento de Ciências Florestais e da Madeira, Universidade Federal do Espírito Santo - UFES, Avenida Governador Carlos Lindenberg, 316, Centro, CEP 29550-000, Jerônimo Monteiro, ES, Brasil

e-mail: djeison.batista@ufes.br

\section{APOIO FINANCEIRO}

Conselho Nacional de Desenvolvimento Científico e Tecnológico (CNPq), bolsa concedida ao primeiro autor. 


\section{REFERÊNCIAS}

Andrade A, Jankowsky IP, Ducatti MA. Grupamento de madeiras para secagem convencional. Scientia Forestalis 2001; 59: 89-99.

Barbosa CG, Lima JT, Rosado SCS, Trugilho PF. Elaboração de programa de secagem para madeiras de clones de híbridos de Eucalyptus spp. Cerne 2005; 11(1): 40-48.

Batista DC, Klitzke RJ, Rocha MP. Proposta de programa de secagem para a madeira de "guajará" (Micropholis venulosa Mart. et Eichler) Pierre, Sapotaceae. Ciência da Madeira 2012; 3(1): 22-32. http://dx.doi.org/10.12953/2177-6830. v03n01a03.

Brandão ATO. Determinação de metodologia para a indicação de programas de secagem de madeiras [dissertação]. Piracicaba: Escola Superior de Agricultura Luiz de Queiroz, Universidade de São Paulo; 1989.

Ciniglio G. Avaliação da secagem de madeira serrada de E.grandis e E.urophylla [dissertação]. Piracicaba: Escola Superior de Agricultura Luiz de Queiroz, Universidade de São Paulo; 1998.

Galvão APM, Jankowsky IP. Secagem racional da madeira. São Paulo: Nobel; 1985

Klitzke RJ, Batista DC. Ensaio de taxa de secagem e escore de defeitos para a predição da qualidade de secagem convencional da madeira de Eucalyptus. Scientia Forestalis 2010; 38(85): 97-105.

Mattos PP, Pereira JCD, Schaitza EG, Baggio AJ. Caracterização física, química e anatômica da madeira de Terminalia ivorensis. Circular Técnica da Embrapa Florestas 1999; 31: 1-14.

Mellado ECR. Contribuição ao desenvolvimento tecnológico para a utilização de madeira serrada de Eucalyptus grandis (Hill ex Maiden) na geração de produtos com maior valor agregado [dissertação]. Curitiba: Setor de Ciências Agrárias, Universidade Federal do Paraná; 1993.

Santos GRV. Otimização da secagem da madeira de Eucalyptus grandis [Hill ex Maiden] [dissertação]. Piracicaba: Escola Superior de Agricultura Luiz de Queiroz, Universidade de São Paulo; 2002.

Severo ETD. Estudo sobre o efeito da vaporização nas propriedades e comportamento de secagem da madeira de Eucalyptus dunnii Maid [tese]. Curitiba: Setor de Ciências Agrárias, Universidade Federal do Paraná; 1998.

Spiegel MR. Estatística. 3. ed. São Paulo: Pearson Education do Brasil/ Makron Books; 1994.

Terazawa S. Methods for easy determination of kiln drying schedule of wood. Japan Wood Industry 1965; 20(5): 216226. [Cited from a translation by H. Sumi into English of the original article written in Japanese.] 